Automatic determination of isostatics in two-dimensional photoelasticity

This article has been downloaded from IOPscience. Please scroll down to see the full text article.

2000 Meas. Sci. Technol. 11259

(http://iopscience.iop.org/0957-0233/11/3/313)

View the table of contents for this issue, or go to the journal homepage for more

Download details:

IP Address: 147.96.14.16

The article was downloaded on 14/05/2013 at 17:45

Please note that terms and conditions apply. 


\title{
Automatic determination of isostatics in two-dimensional photoelasticity
}

\author{
Juan Antonio Quiroga $†$ and Agustín González-Canoł \\ $\dagger$ Centro de Investigaciones en Optica, Loma del Bosque 115, Col. Lomas del Campestre, \\ 37150 Leon, Guanajuato, Mexico \\ \$ Departamento de Óptica, Facultad de Ciencias Físicas, Universidad Complutense, \\ Ciudad Universitaria s/n, 28040 Madrid, Spain \\ E-mail: aq@fis.ucm.es (J A Quiroga) and agus@fis.ucm.es (A González-Cano)
}

Received 17 September 1999, in final form and accepted for publication 16 December 1999

\begin{abstract}
We present a method for automatic determination of isostatics from the photoelastic measurement of isoclinics obtained by phase-shifting techniques. The method is based on the integration of two difference equations by use of a multigrid algorithm, using a quality map obtained from experimental data by checking the consistency of the generated experimental differences. So-called isostatic surfaces are determined and, from them, the isostatics net can be drawn with any prescribed density and resolution. The algorithm is very robust and fast and has proven very efficient for working with real photoelastic fringe patterns.
\end{abstract}

Keywords: photoelasticity, isostatics, phase shifting, multigrid, fringe-pattern analysis

\section{Introduction}

Photoelasticity is a well-established technique for determining stress distributions, on the basis of measurement of the stress-induced birefringence of the model to be tested [1]. In recent years several different methods for evaluating the photoelastic fringe patterns have been developed. They are based on phase-sampling procedures [1-5], Fouriertransform analysis [6] and analysis of spectral contents [7-9], for example.

When an object is subjected to a three-dimensional stress field, the stresses induced at each point of the body can be represented by a symmetrical second-order tensor. This stress tensor can therefore be diagonalized; its principal directions, which are mutually perpendicular, give the principal planes of the state of stress at the point considered. The eigenvalues of the stress tensor correspond to the stresses referred to these principal directions and are called principal stresses. These principal stresses are pure compression or extension, so there is no shear stress in the reference system given by the principal directions. For an elastically deformed body, the stress and the induced birefringence tensors have the same principal directions, so the stress and birefringence can be inferred from the presence of the other.

According to this, in the two-dimensional case, the principal birefringence axes of the model coincide with the principal axes of the two-dimensional state of stress. The retardation $\delta$ induced in a thickness $d$ of the model is

$$
\delta=S\left(\sigma_{1}-\sigma_{2}\right) d
$$

where $\sigma_{1}$ and $\sigma_{2}$ are the two principal stresses and $S$ is the stress-optical constant of the material of the model.
The basic experimental set-up for photoelastic stress analysis is the so-called polariscope. The polariscope has basically two different configurations, planar and circular [1]. A planar polariscope consists of a pair of linear polarizers and the model is inserted between them. A circular polariscope is obtained from the planar polariscope by introducing two quarter-wave plates, the first one after the polarizer and the second before the analyser. The sample is placed between the quarter-wave plates.

When a stressed model is observed with a polariscope, the spatially variable induced retardation and orientation of the principal directions produce a fringe pattern similar to an interferogram. The loci of points where the principal stresses have the same inclination correspond to dark fringes, which are called isoclinics, whereas the loci of points for which the retardation $\delta$ is constant are called isochromatics.

Then, by measuring and evaluating the isochromatic fringe pattern, we can determine the retardation (1) and the difference of principal stresses in every point of the sample. On the other hand, by evaluating the isoclinic fringe pattern, we can determine the local orientation of the principal directions [1].

The stress trajectories are directly related to the isoclinics. A stress trajectory, line of principal stress; or isostatic is a line whose direction at any point coincides with that of one of the principal stresses at the point. Since the two principal stresses at any point of a two-dimensional stress system are mutually perpendicular, it follows that a system of isostatics will consist of two orthogonal families of curves. One of these families indicates the directions of the $\sigma_{1}$ principal stresses and the other indicates those of the $\sigma_{2}$ stresses. Isostatics have been widely used in the analysis 
of photoelastic experiments and are a very useful means of visualizing the behaviour of a given model.

To draw isostatics, there are manual graphic methods [10] and semi-automatic methods in which the user clicks with the mouse for a point and the system draws a cross with the inclination given by the isoclinic parameter. Drawing the isostatic by use of these methods is tedious.

In this work we present an automatic method for drawing isostatics. It is based on the integration of two difference equations obtained from the isoclinic map calculated by any of the techniques referenced above. As we will show, the method is very robust and simple. No starting point needs to be selected and, once the difference equation is solved, the spatial resolution of the isostatics net can be changed in an arbitrary way. Also, the technique can deal with complicated samples containing isotropic points.

This work is organized as follows. In section 2 the theoretical foundations of the method are presented. The integration algorithm used to solve the difference equations is explained in section 3. Experimental results are given in section 4. Finally, conclusions are given in section 5 .

\section{The determination of isostatics from isoclinics}

As we mention above, there exist in the literature several phase-sampling-based strategies for computing isoclinics. They are used with a linear or a circular configuration of the polariscope. By making an appropriate choice of the orientations of the elements of the polariscope, several images are acquired (say, $I_{i}$ and $I_{j}$ ) and then the sine and cosine of the isoclinics angle are computed as

$$
\begin{aligned}
& \sin (K \alpha)=\sum_{i} I_{i} \\
& \cos (K \alpha)=\sum_{j} I_{j}
\end{aligned}
$$

where $K$ is a constant that depends on the algorithm used, whose value can be either 2 or 4 , and $\alpha$ represents the angle of the fast axis of the birefringent sample with respect to one of the reference axes, say $X$. From the sine and the cosine terms the isoclinic angle is computed as

$$
K \alpha=\arctan \left(\frac{\sum_{i} I_{i}}{\sum_{j} I_{j}}\right) .
$$

Because of the arctangent function, the value of $K \alpha$ is determined modulo $2 \pi$. If a circular polariscope in its planar configuration is used, $K$ equals 4 and what we measure by (4) is the angle $\alpha$ modulo $\pi / 2$. The practical consequence of this is that, in the isoclinic map, every time that $\alpha$ reaches a value of $\pm(2 m-1) \pi / 4 \mathrm{rad}$, with $m$ an integer, a sudden jump of value $\pi / 2 \mathrm{rad}$ appears. Thus, the final step in determining $\alpha$ over the area of interest is to remove these jumps. This process is known as phase unwrapping and its result is called a continuous isoclinic map. An example of such a measurement is given in figure 1. Figure 1(a) shows the isoclinic angle $\alpha$ modulo $\pi / 2$ for a disc under diametrical compression; figure $1(b)$ shows the unwrapped isoclinic angle. As expected for this sample, the isoclinic angle is nearly zero in the vertical and horizontal diameters,

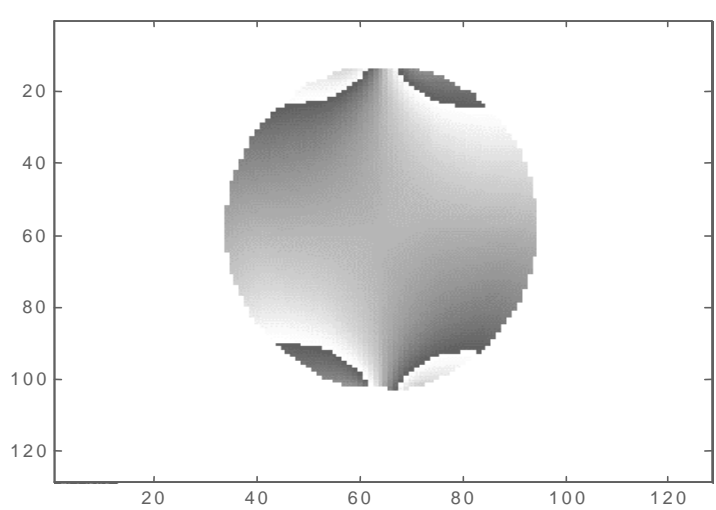

(a)

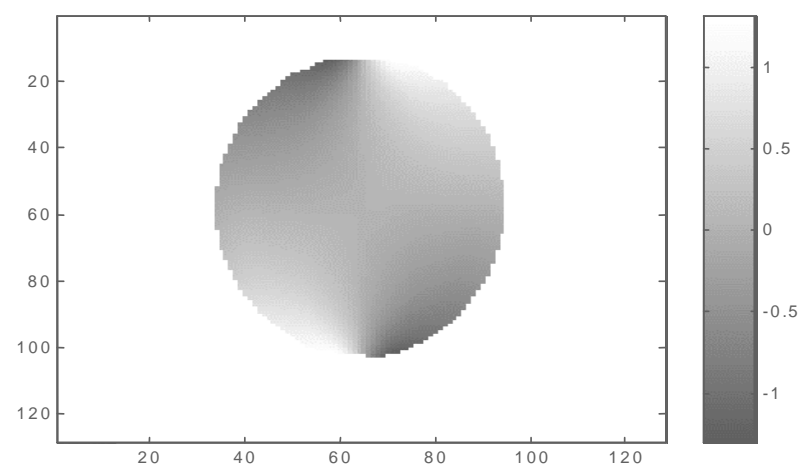

(b)

Figure 1. (a) An isoclinic phase map, $\alpha$, corresponding to a disc under diametral compression. Black represents $-\pi / 4 \mathrm{rad}$ and white represents $\pi / 4$ rad. (b) Demodulation of the phase map shown in $(a)$; the scale is in radians.

whereas it increases towards $\pm \pi / 2$ as we approach the zone of the contact points.

Once the continuous isoclinic map is computed, the determination of the isostatics is straightforward; the only thing that one must do is select a starting point and from it draw a continuous curve, point by point, whose local tangent matches the corresponding isoclinic angle. This is the approach adopted by Almeida and Moitinho [11], who applied this technique to finite-element calculations of plane systems. However, this procedure has some problems. First, the starting points must be selected by the user in such a way that the geometrical information shown by the isostatic net is useful. Second, in real photoelastic data, there is always some amount of noise that can make the process of local line drawing unstable, especially in the surroundings of an isotropic point where the stress tensor is nearly isotropic and thus the isoclinic angle is not well defined.

The approach that we propose is a global procedure based on the following properties of the isostatics [10]: (i) isostatics of one family never intersect each other or merge with those of the other family and (ii) in doubly or multiply connected plates such as rings, or in plates where loads or thermal stresses act within the field of the plate as well as at the edges, the isostatics may form closed loops but not spirals.

This means that we can consider every family of isostatics as contours of a surface and that this surface will always exist, even in the presence of isotropic points, as we will show in section 4 . Thus, if we are to determine the 


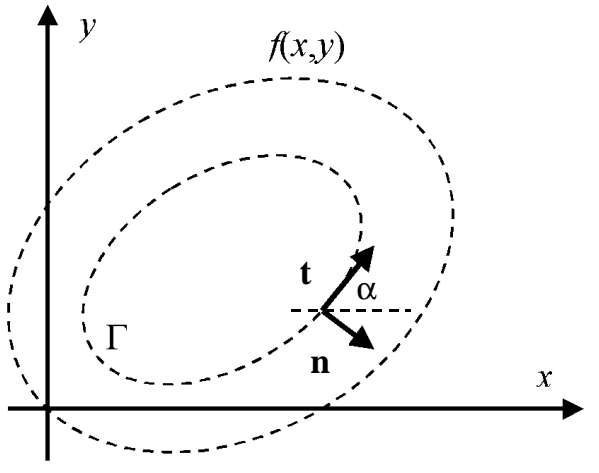

Figure 2. The scheme for the computation of the gradient of the isostatic function $f(x, y)$. If $\alpha$ is the local isoclinic angle and we consider the isostatic $\Gamma$ as a level curve of $f(x, y)$, its gradient, $\nabla f(x, y)$, must be orthogonal to the tangent vector $\boldsymbol{t}=(\cos \alpha, \sin \alpha)$.

corresponding surface for each family of isostatics in a robust and global way, we will have solve the problems depicted above.

If we denote by $z=f(x, y)$ the surface we are looking for and we consider the projection of one of the contour lines of the surface in the $X-Y$ plane, it forms a plane curve denoted by $\Gamma$, according to figure 2 . Taking into account the definition of an isostatic, the unitary tangent vector $t$ at every point of $\Gamma$ fulfils

$$
t=(\cos \alpha, \sin \alpha)
$$

and the unit normal vector must be

$$
\boldsymbol{n}=(\sin \alpha,-\cos \alpha) .
$$

On the other hand, since $\Gamma$ is a contour line, the gradient of $f(x, y)$ is perpendicular to $t$; that is,

$$
\nabla f(x, y) \cdot t=0 .
$$

The simplest solution of (7) is

$$
\nabla f(x, y)=\boldsymbol{n}=(\sin \alpha,-\cos \alpha) .
$$

Then, by solving the difference equation (8), we will find the surface $f(x, y)$ whose contour lines correspond to one family of isostatics.

Following this, the corresponding orthogonal family of isostatics can be derived from a surface $z=g(x, y)$ whose gradient is orthogonal to that of $f(x, y)$; that is,

$$
\nabla g(x, y) \cdot \boldsymbol{n}=0
$$

and the simplest solution to (9) is

$$
\nabla g(x, y)=t=(\cos \alpha, \sin \alpha) .
$$

Then, the solution of (10) is the surface whose contour lines are the second family of isostatics.

Thus, by integrating difference equations (8) and (10), we can obtain the isostatics from the isoclinics. We will call $f(x, y)$ and $g(x, y)$ isostatic surfaces and will refer to equations (8) and (10) as isostatic equations.

However, a remark must be made if an isotropic point is present in the region of interest (ROI). An isotropic point is a place where the difference between the principal stresses is zero, which means that, at that location, the material behaves as if it were isotropic, thus producing a singularity in the continuous isoclinic map $\alpha$. Physically, a first-order isotropic point is a place where the isoclinic angle runs form 0 to $\pi \mathrm{rad}$ as the point is surrounded, for a second-order isotropic point the isoclinic angle runs form 0 to $2 \pi \mathrm{rad}$ and so on. The meaning of this is that, if an isotropic point is present, the $\alpha$ map is not continuous but rather presents a dislocation of $\pm k \pi \mathrm{rad}, k$ being an integer, starting at the isotropic point. In this case the isoclinic map is discontinuous. It can also be the case that the associated functions $f(x, y)$ and $g(x, y)$ given by (8) and (10) will be discontinuous too. Then, the integration algorithm used to solve the isostatic equations must be able to handle this problem, as well as the usual problems of noise and processing of irregular regions of interest.

\section{Integration of the isostatic equations}

The method we use to solve the isostatic equations (8) and (10) is a multigrid algorithm [12]. Multigrid algorithms are methods of solving partial differential equations on large grids, using on the idea of applying Gauss-Seidel relaxation schemes on coarser smaller grids. For the sake of clarity we are going to focus on the solution of isostatic equation (8), but the exactly same procedure applies to isostatic equation (10).

From the experimental photoelastic data $\alpha$ we can obtain an estimation of the discrete partial derivatives of the isostatic surface, $f(x, y)$ with respect to $x$ and $y$, that we will denote by $\Delta_{i j}^{x}$ and $\Delta_{i j}^{y}$. They are given by

$$
\begin{gathered}
\Delta_{i j}^{x}=\sin \alpha \\
\Delta_{i j}^{y}=-\cos \alpha
\end{gathered}
$$

where $i$ and $j$ are the indices that correspond to the columns and the rows of the image, respectively.

The calculation is usually performed only over an area of interest defined by a mask. Here, this mask is supposed to be given and to have been used previously in the computation of the isoclinic angle. This processing mask is defined by a set of binary weights $w_{i, j}^{P}$, which equal 1 inside the ROI and 0 outside it.

Then, our goal is to obtain a set of values $f_{i j}$, whose first partial differences best fit $\Delta_{i j}^{x}$ and $\Delta_{i j}^{y}$ in some sense, taking into account that they are valid only within the ROI defined by $w_{i, j}^{P}$. One possibility is to use the weighted least-squares algorithm. This consists of finding the $f_{i j}$ that minimizes

$$
U=\sum_{i, j} w_{i, j}^{x}\left(D_{x} f_{i j}-\Delta_{i j}^{x}\right)^{2}+w_{i, j}^{y}\left(D_{y} f_{i j}-\Delta_{i j}^{y}\right)^{2}
$$

where $D_{x}$ and $D_{y}$ are the first-difference operators defined as

$$
\begin{aligned}
& D_{x} f_{i j}=f_{i j}-f_{i-1, j} \\
& D_{y} f_{i j}=f_{i j}-f_{i, j-1}
\end{aligned}
$$

and

$$
w_{i j}^{x}=w_{i j}^{y}=w_{i j}^{P}
$$


It can be shown [12] that the $f_{i j}$ that minimizes $U$ can be found by the recursion

$$
\begin{aligned}
f_{i j}= & \frac{1}{v_{i j}}\left[\left(w_{i+1, j}^{x} f_{i+1, j}+w_{i j}^{x} f_{i-1, j}\right.\right. \\
& \left.\left.+w_{i, j+1}^{y} f_{i, j+1}+w_{i j}^{y} f_{i, j-1}\right)-\rho_{i j}\right]
\end{aligned}
$$

with

$$
\begin{gathered}
v_{i j}=w_{i+1, j}^{x}+w_{i j}^{x}+w_{i, j+1}^{y}+w_{i j}^{y} \\
\rho_{i j}=w_{i+1, j}^{x} \Delta_{i+1, j}^{x}-w_{i j}^{x} \Delta_{i j}^{x}+w_{i, j+1}^{y} \Delta_{i, j+1}^{y}-w_{i j}^{y} \Delta_{i j}^{y} .
\end{gathered}
$$

It is easy to see that (15) is a weighted version of the wellknown Gauss-Seidel relaxation. One of the characteristics of the Gauss-Seidel relaxation is that it obtains the highfrequency details very fast, but low-frequency features are difficult to obtain, since in each step of the iteration every point receives information only on its immediate neighbours.

In multigrid methods the key idea is to transform the lowfrequency components of the solution obtained by the GaussSeidel relaxation in a fine grid into high-frequency components of a coarser grid, where Gauss-Seidel relaxation works well. Afterwards, we need only translate the results obtained to the finer grids. A set of grids, each with a double spacing between points, can be used and the information must be transferred back and forth between fine and coarse grids. This is accomplished by two operations, prolongation (that extends the results obtained with coarse grids to fine grids) and restriction (that performs the opposite operation, namely, the transfer of the information from the fine to the coarse grid). For this reason, multigrid methods are especially well suited for improving the efficiency of the Gauss-Seidel relaxation scheme represented by (15). The specific details of the multigrid algorithm and the forms of the prolongation and restriction operators, as well as a code listing, can be found in [12].

This procedure works well if there are no isotropic points in the ROI. As we explained above, if an isotropic point is present inside the ROI, the isoclinic map is discontinuous; thus the resulting isostatic surface can be discontinuous too. For the least-squares formulation this is a big problem, so we must design a mask to prevent the integration algorithm from passing over these discontinuities, if they exist.

The way we determine this mask is based on the consistency of the pair of differences (11). A pair of differences is said to be consistent if the integral around every simple closed path is zero. In a regular grid the simplest closed path is a $2 \times 2$ square and the result of performing the test of consistency is assigned to the upper-left pixel. According to this, the consistency of the pairs of differences is computed from (11) as

$$
C_{i j}=w_{i j}\left(\Delta_{i, j}^{x}+\Delta_{i+1 j}^{y}-\Delta_{i, j+1}^{x}-\Delta_{i j}^{y}\right)
$$

where $w_{i j}=\min \left(w_{i j}^{P}, w_{i+1, j}^{P}, w_{i, j+1}^{P}\right)$.

In phase unwrapping, wherein the first differences are computed from the phase data, the values for $C_{i j}$ can only be either 0 or \pm 1 , depending on whether a source of discontinuity is present. In our case, $C_{i j}$ can have in principle any value. So, more than just being a mask telling us whether a point is valid, the consistency (18) provides us with a quality map that tells us how good the pair of differences at the point considered is; $\left|C_{i j}\right|=0$ indicates a perfect match. Even if

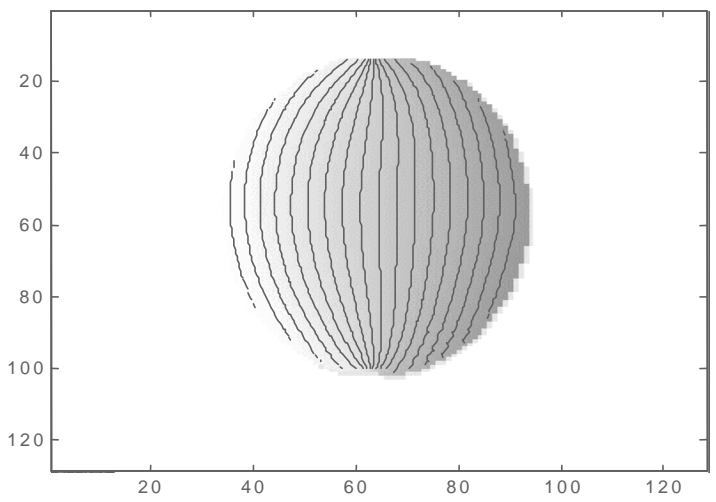

(a)

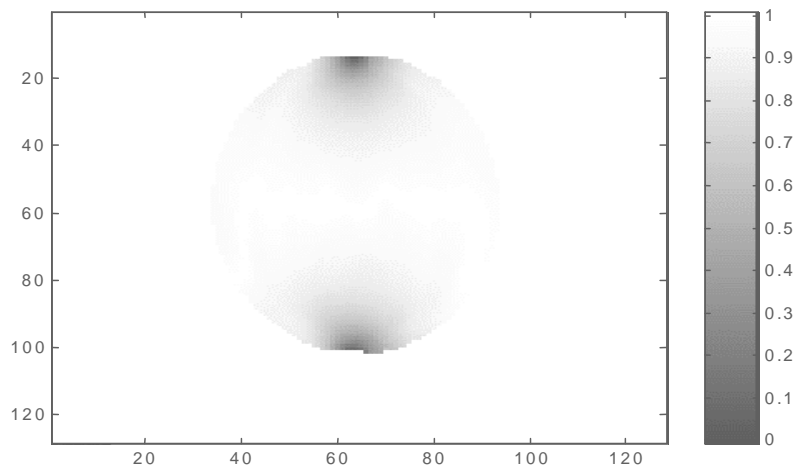

(b)

Figure 3. (a) The isostatic surface $f(x, y)$ corresponding to the isoclinic map of figure 1 . The lines are the level curves of $f(x, y)$, that is, the first family of isostatics. (b) The consistency map, $w_{i j}^{C}$, corresponding to the differences used to integrate the isostatic surface $f(x, y)$ shown in $(a)$; in this image the lower grey values correspond to the smaller values of the consistency map.

there is no isotropic point in the ROI, there is always a lack of consistency near the contact points or due to the noise. Taking this into account, we can define a quality map based on the consistency as

$$
w_{i j}^{C}=1-\frac{\left|C_{i j}\right|}{\max \left(\left|C_{i j}\right|\right)}
$$

and, from its definition, $0 \leqslant w_{i j}^{C} \leqslant 1$. Much better results are obtained if, prior to the computation of $w_{i j}^{C}$, the values of $\left|C_{i j}\right|$ above a certain threshold are set to the maximum of $\left|C_{i j}\right|$, producing in this way a zero weighting in $w_{i j}^{C}$, thus avoiding the processing of the points marked by these zero weights. In all the cases processed by us, a value of 0.5 for this threshold works well.

Finally, the weights for the integration of the pair of differences (11) by means of the minimization of (12) are given by

$$
w_{i j}^{x}=w_{i j}^{y}=w_{i j}^{P} w_{i j}^{C}
$$

where the processing mask has been combined with the consistency quality map. For the case of the isostatic surface $g(x, y)$, the processing is the same, but in this case the differences to be integrated are

$$
\begin{aligned}
\Delta_{i j}^{x} & =\cos \alpha \\
\Delta_{i j}^{y} & =\sin \alpha .
\end{aligned}
$$




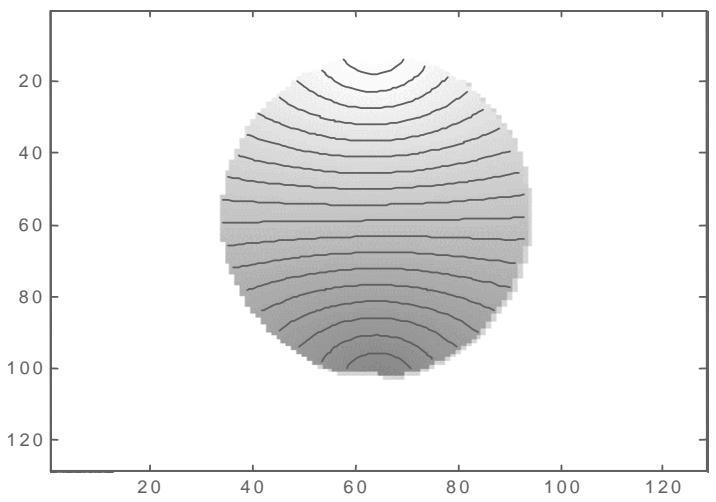

(a)

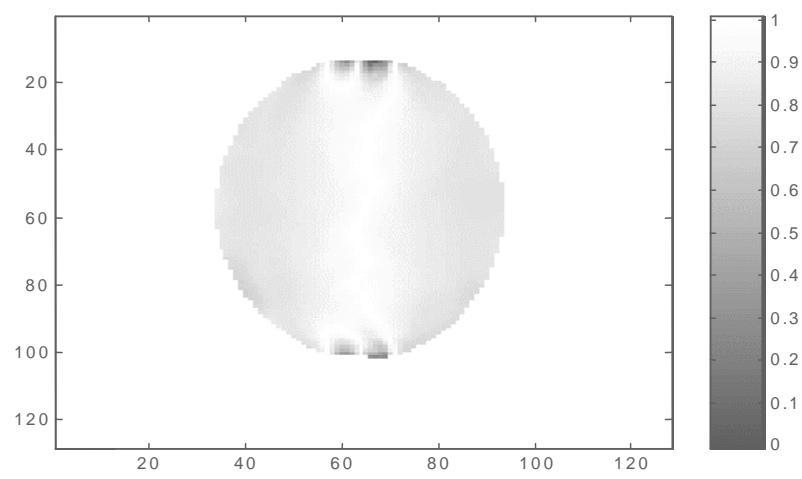

(b)

Figure 4. The isostatic surface $g(x, y)$ corresponding to the isoclinic map of figure 1. The lines are the level curves of $g(x, y)$, that is, the second family of isostatics. (b) The consistency map, $w_{i j}^{C}$, corresponding to the differences used to integrate the isostatic surface $g(x, y)$ shown in $(a)$; in this image the lower grey values correspond to the smaller values of the consistency map.

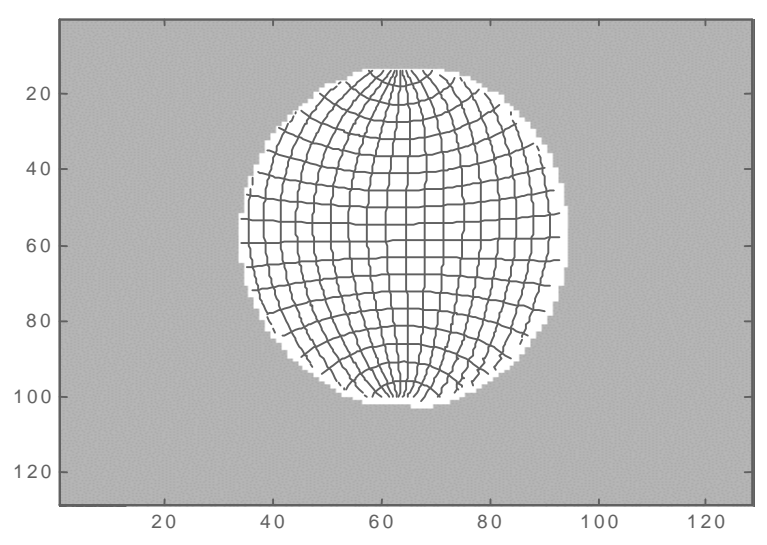

Figure 5. The isostatic net for the loaded disc.

\section{Experimental results}

The first sample is a disc under diametral compression. Figure 1(a) shows the isoclinic angle $\alpha$ modulo $\pi / 2$ for a disc under diametrical compression and figure $1(b)$ shows the unwrapped isoclinic angle. The algorithm used to obtain this map is depicted in [13]. The processing time for that operation is about $20 \mathrm{~min}$. The algorithm automatically identifies the discontinuities associated with isotropic points. In this case there are no isotropic points in the ROI, so the iso-

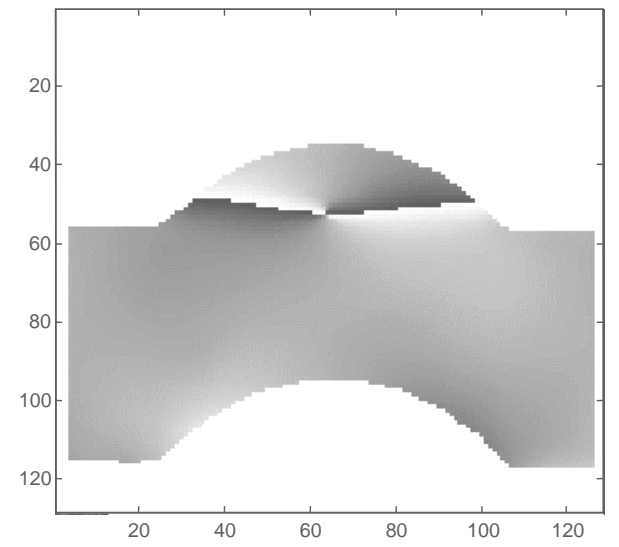

(a)

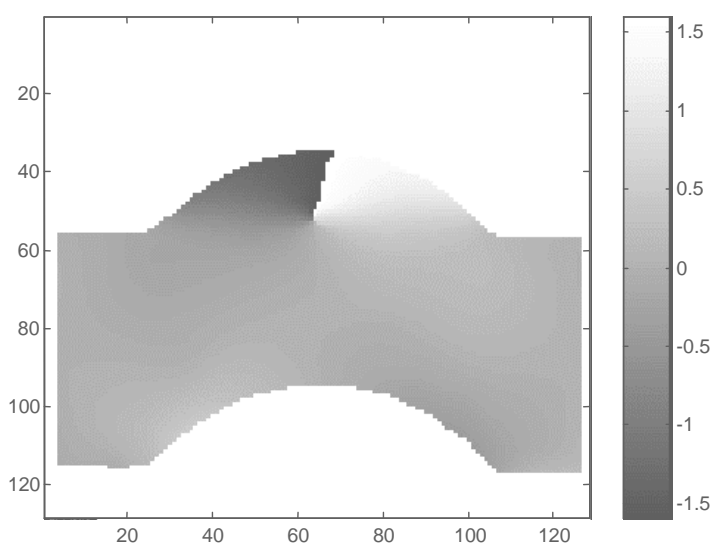

(b)

Figure 6. (a) The isoclinic phase map, $\alpha$, corresponding to the second sample; the force is a traction applied horizontally in the extremes of the bar. In the upper part of the arc an isotropic point is clearly visible as two close positive discontinuities in the phase map. Black represents $-\pi / 4 \mathrm{rad}$ and white represents $\pi / 4 \mathrm{rad}$. (b) Demodulation of the phase map shown in $(a)$; the scale is in radians.

clinic angle is continuous. Figures $3(a)$ and $(b)$ show the isostatic surface $f(x, y)$ and the corresponding consistency map $w_{i j}^{C}$, respectively. As we mentioned above, there is a lack of consistency near the contact points, as figure $3(b)$ shows. In figure $3(a)$, the contours of $f(x, y)$ are superposed on the image, showing the first family of isostatics. Figures $4(a)$ and (b) show the isostatic surface $g(x, y)$ and the corresponding consistency map $w_{i j}^{C}$, respectively. The contours of $g(x, y)$ are superposed on the image, showing the second family of isostatics. Figure 5 is a plot of the isostatics net, as can be seen, the result corresponds to that we expect from a disc under diametrical compression according to the isoclinics map of figure $1(b)$. A feature of our method is that the net spacing can be selected in various ways: according to the gradients of the isostatic surfaces, to their curvature, etc. We have chosen a net spacing so that the isostatics drawn would represent equidistant level curves of the isostatic surface.

The second example is a bar with a curved part loaded in extension along the horizontal direction. Figures $6(a)$ and (b) show the isoclinic angle modulo $\pi / 2$ and the unwrapped isoclinic angle. The isotropic point is located in the upper part of the arc, as can be seen in figure $6(b)$. If we encircle it we will find a dislocation of $\pi \mathrm{rad}$ starting from it and 


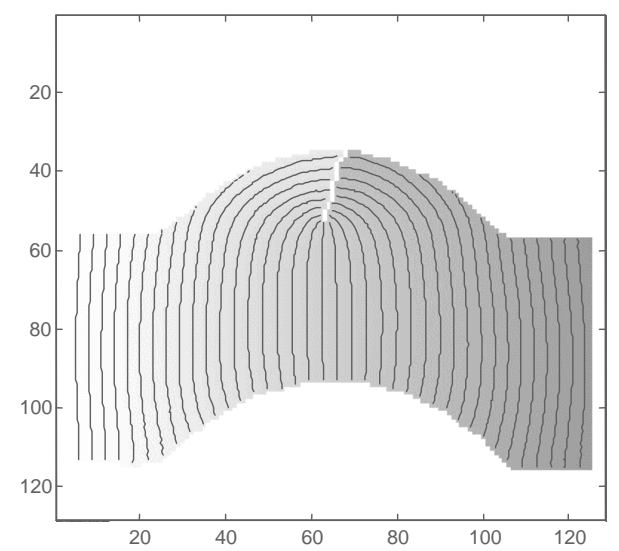

(a)

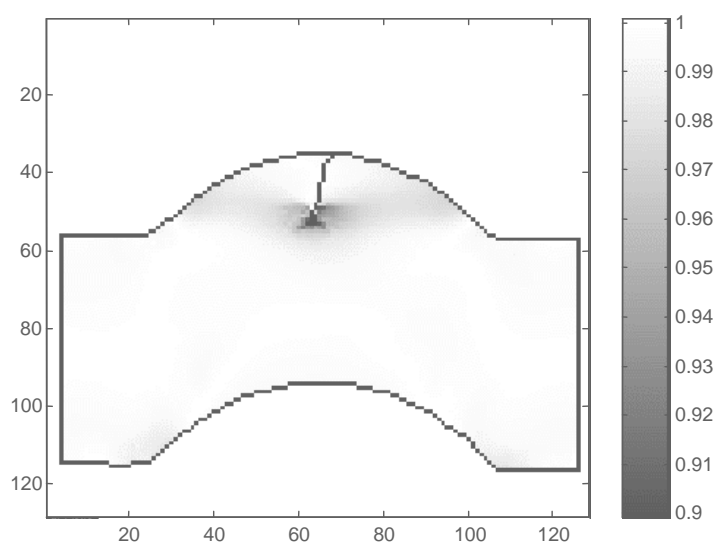

(b)

Figure 7. (a) The isostatic surface $f(x, y)$ corresponding to the isoclinic map of figure 6 . The lines are the level curves of $f(x, y)$, that is, the first family of isostatics. (b) The consistency map, $w_{i j}^{C}$, corresponding to the differences used to integrate the isostatic surface $f(x, y)$ shown in $(a)$; in this image only the values between 0.9 and 1 are shown. It can be seen that the consistency map generates a 'cut line' starting at the isotropic point and ending on the border. The external border of the region of interest is marked for clarity.

directed towards the upper border, so it is a first-order isotropic point. Figures $7(a)$ and $(b)$ show the isostatic surface $f(x, y)$ and the corresponding consistency map $w_{i j}^{C}$, respectively. In this case the consistency map exhibits very low values along the discontinuity of the isoclinic map; thus the resulting isostatic surface is discontinuous, as can be seen in figure 7(a); but even in this case the projections of the contours in the $X-Y$ plane are continuous. This is because, on both sides of the discontinuity, there is a change in the $z$ component of the slope but not in the $x$ and $y$ components. In figure $7(a)$ the contours of $f(x, y)$ are superposed on the image, showing the first family of isostatics. Figures 8(a) and (b) show the isostatic surface $g(x, y)$ and the corresponding consistency map $w_{i j}^{C}$, respectively. In this case, apart from some isolated points over the discontinuity of the isoclinic map, the consistency map has no separation lines like in the example of figure $7(b)$; thus the resulting isostatic surface is continuous. Again, in figure 8(a) the contours of $g(x, y)$ are superposed on the image, showing the second family of isostatics for this case. Finally, in figure $9(a)$ the isochromatic parameter $\delta$ modulo $2 \pi$ is shown with the isostatic net

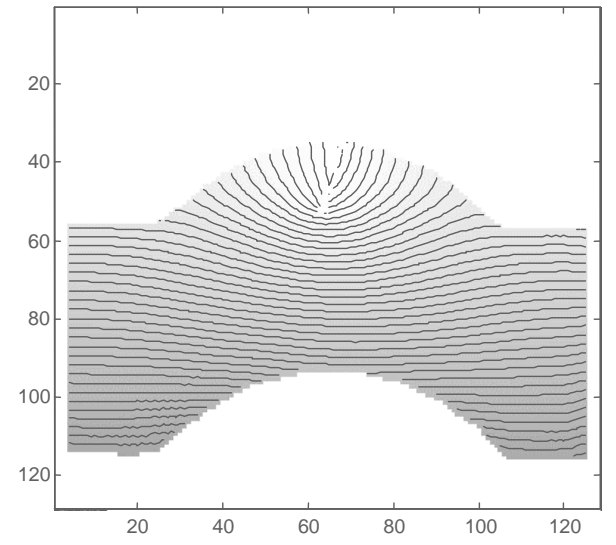

(a)

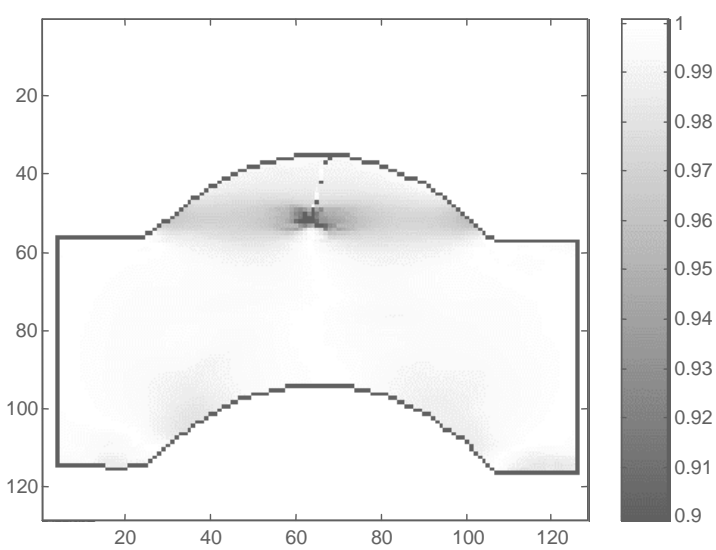

(b)

Figure 8. (a) The isostatic surface $g(x, y)$ corresponding to the isoclinic map of figure 6 . The lines are the level curves of $g(x, y)$, that is, the second family of isostatics. (b) The consistency map, $w_{i j}^{C}$, corresponding to the differences used to integrate the isostatic surface $g(x, y)$ shown in $(a)$. In this image only the values between 0.9 and 1 are shown. The external border of the region of interest is marked for the sake of clarity.

superposed. In figure $9(a)$ the isotropic point is easily identifiable as a positive isotropic point (because of the interlocking isostatic net). Also, it can be observed that the free boundary is an isostatic except when the boundary stresses change in sign; at these points the parallel isostatic changes its course and becomes normal, while the normal isostatic becomes parallel. This phenomenon occurs at the points marked by arrows in figure $9(a)$, where the stress boundaries change in sign from extension to compression. Figure $9(b)$ shows the magnification of the isostatic net corresponding to the rectangle marked in figure $9(a)$; this picture shows graphically the ability to increase the resolution in the drawing of the isostatic net of the present method.

In the two examples shown the size of the images was $128 \times 128$ and the total processing time was a few seconds. To test the performance of the algorithm with a more realistic image, we have used a photoelastic pattern from a turbine model in a frozen-stress state. The results are shown in figure 10, in which the isostatics net is shown superposed on the isochromatic map. The size of the image was $512 \times 512$ pixels and the processing time was $60 \mathrm{~s}$ with a $300 \mathrm{MHz}$ Pentium PC. 


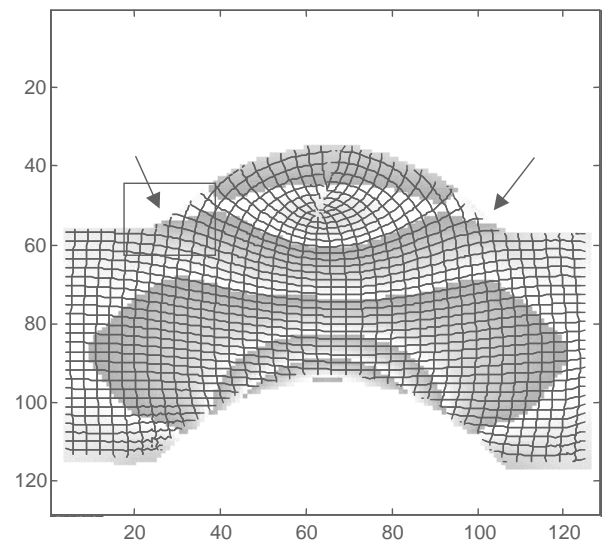

(a)

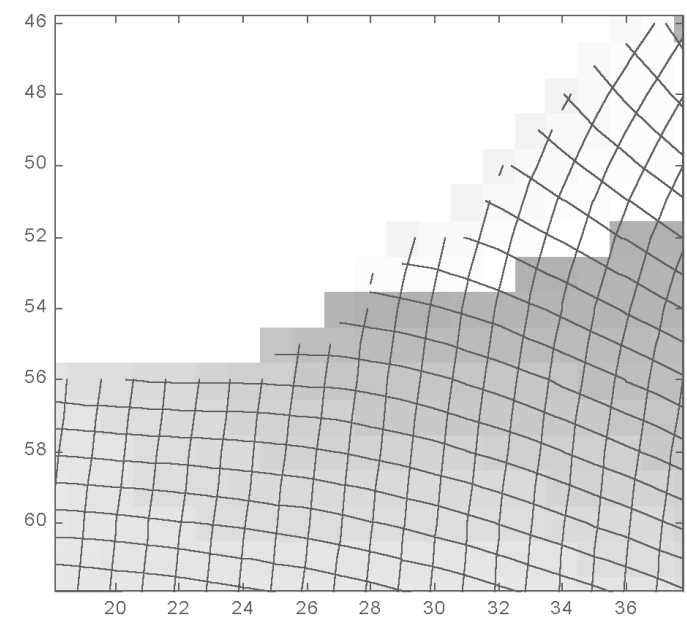

(b)

Figure 9. (a) The isostatic net of the second sample superposed on the isochromatic phase map $\delta$. In the phase map black levels indicate 0 and white indicates $2 \pi$ rad. In this figure it is clear that there is an interlocking system of isostatics, indicating that a positive isotropic point is present. The arrows indicate the positions where boundary stresses change in sign. (b) A magnification of the region marked by the rectangle in $(a)$; in this image it is clearly visible how the isostatics parallel to the border became perpendicular and vice versa, indicating a change in sign of the boundary stresses.

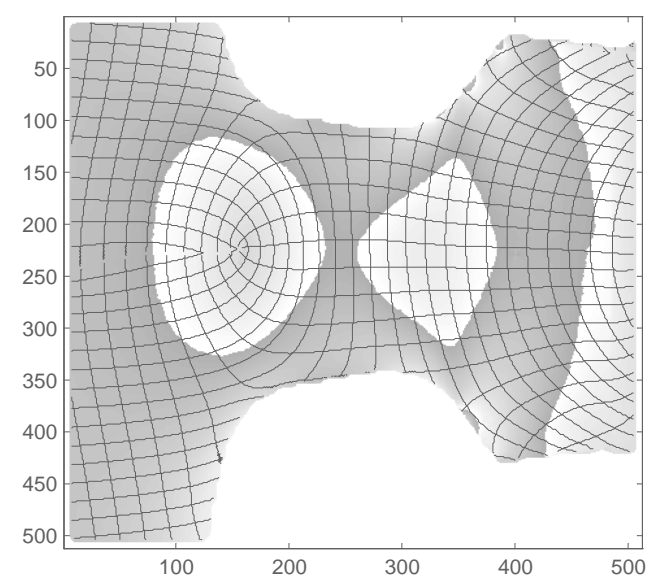

Figure 10. The isochromatic map and isostatics net for a turbine model in a frozen-stress state. In the phase map black levels indicate 0 and white indicates $2 \pi \mathrm{rad}$.

\section{Conclusions}

We have presented a method for automatic determination of isostatics from the photoelastic measurement of isoclinics. The method is based on the integration of two difference equations generated from the isoclinic angle. To integrate these difference equations we have used a multigrid algorithm with a quality map computed from the experimental data by checking the consistency of the experimental differences generated. The method is robust and reliable and the use of the multigrid algorithm allows short processing times and realistic image sizes. The method does not depend on the selection of any starting point. Once the so-called isostatics surfaces are computed, the isostatic net can be drawn with prescribed density and resolution. Also, the algorithm can deal with problems associated with isotropic points. The method has been proved to be well suited to processing real photoelastic problems.

\section{Acknowledgments}

We wish to acknowledge the support of a post-doctoral grant from the Universidad Complutense de Madrid, the 'Becas Internacionales Flores-Valles' program, Spain, and the Centro de Investigaciones en Óptica (CIO), León, Mexico. This work has been partially supported by the project TAP 98 0701/97 of the CICyT (Comision Interministerial de Ciencia y Tecnologia).

\section{References}

[1] Theocaris P S and Gdoutos E E 1979 Matrix Methods in Photoelasticity (Berlin: Springer)

[2] Asundi A 1993 Phase shifting in photoelasticity Exp. Techn. 17 19-23

[3] Petrucci G 1997 Full-field automatic evaluation of an isoclinic parameter in white light Exp. Mech. 37 420-6

[4] Quiroga J A and González-Cano A 1997 Phase measuring algorithm for extraction of isochromatics of photoelastic fringe patterns Appl. Opt. 36 8397-402

[5] Plouzenec N, Dupré J C and Lagarde A 1999 Whole field determination of isoclinic and isochromatic parameters Exp. Techn. January/February 30-3

[6] Morimoto Y, Morimoto Y and Hayashi T 1994 Separation of isochromatics and isoclinics using Fourier transform Exp. Techn. September/October 13-17

[7] Carazo-Alvarez J, Haake S J and Patterson E A 1994 Completely automated photoelastic fringe analysis $O p t$. Laser Engng. 21 133-49

[8] Buckberry C and Towers D 1995 Automatic analysis of isochromatic and isoclinic fringes in photoelasticity using phase-measuring techniques Meas. Sci. Technol. 6 1227-35

[9] Nurse A D 1997 Full-field automated photoelasticity by use of a three-wavelength approach to phase stepping Appl. Opt. 36 5781-6

[10] Kuske A 1974 Photoelastic Stress Analysis (New York: Wiley)

[11] Almeida-Pereira O J B and Moitinho de Almeida J P B 1994 Automatic drawing of stress trajectories in plane systems Computers Structures 53 473-6

[12] Ghiglia D C and Pritt M D 1998 Two-Dimensional Phase Unwrapping: Theory, Algorithms, and Software (New York: Wiley)

[13] Quiroga J A and González-Cano A 2000 Separation of isoclinics and isochromatics from photoelastic data using a regularized phase-tracking technique Appl. Opt. to appear 